\title{
Decoding cancer metastasis
}

\author{
Understanding the dynamics and complexity of tumour metastasis is crucial for improving clinical interventions \\ and care for cancer patients. In this issue, we present the first of a Series of commissioned Review articles that \\ discuss emerging concepts, technological advances and therapeutic implications in this exciting field.
}

C ancer is a constantly evolving disease with extraordinary heterogeneity at multiple levels. Distinct cancer types exhibit vastly heterogeneous oncogenic alterations, and this extends to cancer cells of the same tumour, which can exhibit variability in genetic, transcriptomic and epigenetic signatures. Added to this is the heterogeneous composition of the tumour microenvironment, which comprises distinct non-tumour cell types, signalling molecules, nutrient levels and stroma composition. This tumour cell-intrinsic and -extrinsic diversity is exacerbated by context-dependent factors at the organismal and environmental level; tumours of the same histological category may lead to different clinical outcomes in different patients. This extraordinary complexity thus leads to heterogeneity in the clinical setting, such as distinct dynamics in tumour progression and metastatic organotropism.

The metastatic cascade is itself highly complex, starting with invasion of primary tumour cells into the surrounding tissue, continuing as intravasation into the circulation and extravasation to a distant organ, where disseminated tumour cells that survive may initiate the growth of a secondary tumour in a metastasis-receptive niche. This cascade involves overcoming physical barriers, physiological stress and host defence mechanisms, and incurs substantial energy demands, thus making it a highly inefficient process. Disseminated tumour cells must adopt effective survival and adaptation strategies to migrate to and thrive in an incompatible distant microenvironment.

Metastasis remains one of the main reasons for the poor prognosis of cancer patients. The Surveillance, Epidemiology and End Results (SEER) Program hosted by the National Cancer Institute (https://seer.cancer. gov/statfacts/) reveals that the 5-year relative survival rate is $98.7 \%$ in female patients with localized breast cancer, but drops to merely $27 \%$ in those with metastasized cancer. These depressing statistics speak to the importance of improving our understanding of the metastatic process to therapeutically constrain the disease and ultimately offer viable cure options to cancer patients.

Given the rapidly evolving knowledge and pivotal clinical implications in this field, we are pleased to launch our Series on Tumour Metastasis. The Series comprises specially commissioned Review articles covering different aspects of the topic, and is accompanied by a selection of related research and comment articles published in Nature Cell Biology over the past two years. Readers may access all content through a dedicated page of our website (https://www.nature.com/ collections/bpfzlylrnw/) that will be updated with new primary and commissioned pieces.

The Series begins with a Review article by Toni Celia-Terrassa and Yibin Kang discussing the pathophysiological function and therapeutic relevance of metastatic niches (Nat. Cell Biol. https://doi.org/ s41556-018-0145-9). Steven Paget's 'seed and soil' hypothesis first introduced the concept of the microenvironment at a distant organ being conducive to engraftment and outgrowth of disseminated tumour cells to form metastatic lesions (Lancet 133, 571-573; 1889). In this landmark work, Paget showed that breast tumour cells preferably metastasized to bones and visceral organs, and concluded that metastasis is not stochastic and not all distant organs are equally receptive to metastatic formation, providing early and crucial evidence that properties of the 'soil' in the distant organs are important determinants of the metastatic outcome. Our understanding of such a metastasis-receptive microenvironment, a metastatic niche, has advanced rapidly in recent years. In particular, colonization of specific metastatic niches by tumour cells is considered as a crucial step during the metastatic process. Celia-Terassa and Kang propose four major common functions of metastatic niches in promoting anchorage, survival, protection and outgrowth of disseminated tumour cells. They also discuss tumour cell plasticity properties and mechanisms, and the interactions of tumour cells with the non-tumour components of metastatic niches, such as immune cells. The Review concludes with outstanding questions and future prospects on how our expanding knowledge of metastatic niches may be leveraged to improve treatment and management for metastatic malignancies.

In the coming months, the Series will focus on other aspects of the metastatic process, such as tumour dormancy.
This phenomenon refers to the clinical observation that disseminated tumour cells remain inert and asymptomatic for lengthy periods of time, often many years, before reinitiating proliferation for metastatic growth. It is estimated that millions of cells are shed from primary tumours per gram mass each day, but only a few become capable of migrating and surviving in a distant organ. Mechanistically, how a quiescent state in these few disseminated tumour cells is induced, maintained and deactivated by environmental cues at cellular and molecular levels remains a critical question to address, particularly given the increasingly recognized importance of the immune system in regulating tumour dormancy.

Despite our improved understanding of tumour progression and metastasis in recent years, the underlying heterogeneity and complexity of the pathological process makes it essential to trace the evolution and progression of the disease from primary tumours to metastatic lesions. Rapid technological developments have now enabled detailed molecular dissection of primary and metastatic tumours in parallel to map disease progression and heterogeneity from a temporal and spatial perspective. This progress is exemplified by the recent advancement of single cell technologies, which have transformed and enriched our understanding of tumour development and progression. Indeed, interrogating bulk tumour masses at the whole population level is not sufficient to thoroughly understand how individual tumour cells behave and affect functional output, such as metastasis and drug response. Single cell approaches have now allowed for high-throughput investigation of tumour heterogeneity and metastasis at an unprecedented level.

Although much remains to be discovered and characterized in this rapidly evolving field, we hope that this Series will bring intriguing concepts and insightful discussion into mechanisms and clinical translation that will stimulate further interrogation of tumour metastasis. We thank our authors and referees for their contributions, and hope that you will find this Series enjoyable and enlightening. $\square$

Published online: 26 July 2018 https://doi.org/10.1038/s41556-018-0167-3 